\title{
Role of afIR gene expression from A. flavus to cause disease in human
}

\begin{abstract}
The aflR gene is a regulatory gene for aflatoxin biosynthesis, encodes a protein containing a zinc-finger DNA-binding motif. There is a positive regulatory gene, aftR which required for transcriptional activation of most, if not all, of the structural genes by binding to the palindromic sequence 5'-TCGN5CGA-3' in the promoter region of the structural genes in A. parasitic, A. flavus and in A. nidulans. Adjacent to the afR gene, a gene, afIS afJJ, is also involved in the regulation of transcription. Finally, the laeA gene, for loss of afR expression, was shown to be involved in the global regulation of secondary metabolites, aflatoxins, sterigmatocystin (ST), penicillin and gliotoxin, in several fungal species. Aflatoxins are polyketide-derived secondary metabolites produced by Aspergillus parasiticus, Aspergillus flavus, Aspergillus nomius and a few other species. The toxic effects of aflatoxins have opposing consequences for the health of human and agricultural economics. This study will examine to understand the mechanisms of afR gene regulation, documentation of transcriptional activation.
\end{abstract}

Keywords: sterigmatocystin, aspergillus parasiticus, aspergillus nomius, aflatoxins, penicillin
Volume 5 Issue 2 - 2017

\author{
Saleem Ahmad, ${ }^{1,2}$ Farman Ali ${ }^{2,3}$ \\ 'Key Laboratory of Pathogenic Fungi and Mycotoxins of Fujian \\ Province, Fujian Agriculture and Forestry University, China \\ ${ }^{2}$ Key Laboratory of Biopesticide and Chemical Biology of \\ Education ministry, and School of Life Science, Fujian Agriculture \\ and Forestry University, China \\ ${ }^{3}$ Key Laboratory of Agroecological Processing and Safety \\ Monitoring, Fujian Agriculture and Forestry University, China
}

\begin{abstract}
Correspondence: Saleem Ahmad, Key Laboratory of Pathogenic Fungi and Mycotoxins of Fujian Province,

Key Laboratory of Biopesticide and Chemical Biology of Educationministry and School of Life Science, Fujian Agriculture and Forestry University, Fuzhou 350002, China, Tel 18859144980,Email saleem.chilas@yahoo.com
\end{abstract}

Received: June 26, 2017 | Published: July 18, 2017

\section{Introduction}

Aspergillus flavus is an opportunistic pathogen. ${ }^{1}$ The genus Aspergillus is a member of the phylum Ascomycota include over 185 known species. There are 20 species of them have been reported to cause harmful infections in humans and animals ${ }^{2}$ causing invasive and non-invasive aspergillosis in humans, animals and insects. It also causes allergic reactions in humans. ${ }^{3}$ A. flavus infects crops, stored grains and produces the most toxic and potent carcinogenic metabolites such as aflatoxins and other mycotoxins. ${ }^{4}$ Aflatoxins are a family of toxins, polyketide-derived metabolites from Aspergillus flavus and Aspergillus parasiticus. ${ }^{5}$ Many aflatoxin pathway genes revealed that are clustered within a $60-\mathrm{kb}$ DNA region in $A$. parasiticus and $A$. flavus. ${ }^{6}$ This finding has renewed interest in the study of the regulation of aflatoxin biosynthesis. ${ }^{5}$ Former studies have suggested that one of these genes i. e. aflR, is involved in some aspect of the regulation of aflatoxin biosynthesis. ${ }^{7}$ The aflR gene product, AFLR, contains a GAL4-type binuclear zinc finger cluster Cys-(Xaa)2-Cys-(Xaa)6-Сys(Xaa)6-Cys-(Xaa)2-Cys-(Xaa)6-Cys. ${ }^{8}$ The(Cys)6-type structure is a distinct feature of some of the pathway-specific regulatory proteins in fungi and yeasts. Also, aflR contains a highly acidic domain adjacent to the carboxyl terminus, ${ }^{9}$ similar to that found in GAL4 and GCN4. In Saccharomyces cerevisiae, this region is necessary for the DNAbinding protein's ability to activate transcription of other pathway related genes. ${ }^{10}$ The transformation with aflR of a mutant of $A$. flavus, which inhibit the production of all aflatoxin pathway precursors and aflR restored it to aflatoxin proficiency. ${ }^{11}$ Wild-type and blocked $A$. parasiticus strains, on transformation with aflR-containing vectors, ${ }^{5}$ over produced aflatoxin precursors in aflatoxin permissive media, such as potato dextrose broth and Adye and Mateles medium. ${ }^{12}$ Aflatoxins or precursors were also produced by these transformants when cells were grown in nitrate medium, which normally inhibits aflatoxin production. ${ }^{13}$ Interestingly, sclerotial morphogenesis was also affected by these afR transformations grown in the nitrate medium.

Nitrogen regulates he biosynthesis of secondary metabolites in a variety of microorganisms. ${ }^{5}$ Nitrate represses the production of polyketide-derived mycotoxins in A. parasiticus and Alternaria alternata. Niehaus suggested that repression by nitrate is due to an increased cytoplasmic NADPH/NADP ratio, which favors a biosynthetic reduction, promoting utilization of malonyl coenzyme A and NADPH for fatty acid synthesis, rather than for polyketide synthesis. $^{7}$ Other researchers suggested that nitrate represses formation of enzymes involved in polyketide synthesis. ${ }^{5}$ In this report, we show that nitrate inhibits transcription of aflatoxin pathway genes in A. parasiticus, including aflR, nor-1, ver-1, and omt-1 but the transcription of these genes is restored in transformants containing an additional copy of aflR. ${ }^{14}$

\section{Fungal Strains and Isolation of Total RNA}

A. flavus, a wild-type aflatoxigenic strain, and SU1-N3(pHSP), a derivative of SU-1 that transformed with the afR-containing vector pHSP, were maintained on Potato Dextrose Agar(PDA). ${ }^{15}$ A. flavus mycelia grows in parallel for 48, 72, 96 and 120h in Cove'sminimal salt medium (CMSM)supplemented with ten $\mathrm{mM}$ nitrate (nitrate medium), ${ }^{5}$ collected on Miracloth, blotted dry, quickly frozen in liquid nitrogen, and stored at $28^{\circ} \mathrm{C}$ until use..$^{11}$ Mycelia is grind to a fine powder in a mortar and pestle in the presence of liquid nitrogen. ${ }^{16}$

\section{Northern (RNA) hybridization analysis}

Twenty micrograms of total RNA per sample fractionated in a $0.4 \mathrm{M}$ formaldehyde $-1.2 \%$ agarose gel and transferred to a Gene Screen Plus membrane (Dupont NEN Research Products, Boston, Mass.), ${ }^{17}$ for probing with [a-32P]dCTP-radiolabeled DNA probes prepared by using the Random Primed DNA Labeling Kit. ${ }^{18}$ 


\section{Cloning and sequencing of afIR cDNA}

A cDNA library in lambda phage vector constructed with a directional ZAP-cDNA synthesis kit. ${ }^{5}$ Screening of the cDNA library performed with [a-32P] dCTP-labeled probes generated from a 1.9-kb BamHI-BamHI genomic DNA fragment In vivo excision of pBluescript SK2 phagemids from positive Uni-ZAP XR lambda clones conducted with the ExAssist/SOLR system (Stratagene) by a helper phage coinfection protocol. Several positive cDNA clones isolated and analyzed by restriction enzyme digestion. ${ }^{19}$ The clone containing the largest cDNA insert sequenced by the dideoxy chain termination method with Sequenase version. ${ }^{5}$ The aflR cDNA sequence including the 59 upstream sequences and the deduced amino acid sequence..$^{20}$ The copy number of the aflR transformants determined by a quantitative slot hybridization technique with a GeneScreen Plus nylon membrane. ${ }^{21}$

\section{Determination of afIR copy number in transformants}

The copy number of the aflR transformants determined by a quantitative slot hybridization technique with a GeneScreen Plus nylon membrane. The SU1-N3 and SU1-N3(pHSP) genomic DNAs denatured in $1 \mathrm{ml}$ of $0.25 \mathrm{M} \mathrm{NaOH}-0.5 \mathrm{M} \mathrm{NaCl}$ for $10 \mathrm{~min}$ at room temperature. Four 200-ml portions of denatured DNA is loaded into individual sample wells of the apparatus.PCR performed by using Ampli Taq DNA polymerase and a DNA thermal cycler., ${ }^{5,11}$

\section{Expression and purification of recombinant afIR}

The SmaI-XhoI digest of aflR cDNA ligated to SmaI-XhoI-digested pET29c (Novagen, Madison, Wis.) to give the plasmid pAFLR1. This construct has in-frame codons for six histidines at the C-terminal side of the aflR insert. The plasmid transformed into Escherichia coli BL21 (DE3) pLysS, and expression is induced by adding isopropylb-D-thiogalactopyranoside (IPTG) to a final concentration of $1 \mathrm{mM}$ to $\log$-phase E. coli cells grown in Luria broth (LB) medium containing kanamycin and chloramphenicol. Expression of the fusion protein is monitored by sodium dodecyl sulfatepolyacrylamide gel electrophoresis (SDS-PAGE) in aliquots taken at hourly intervals. ${ }^{5}$

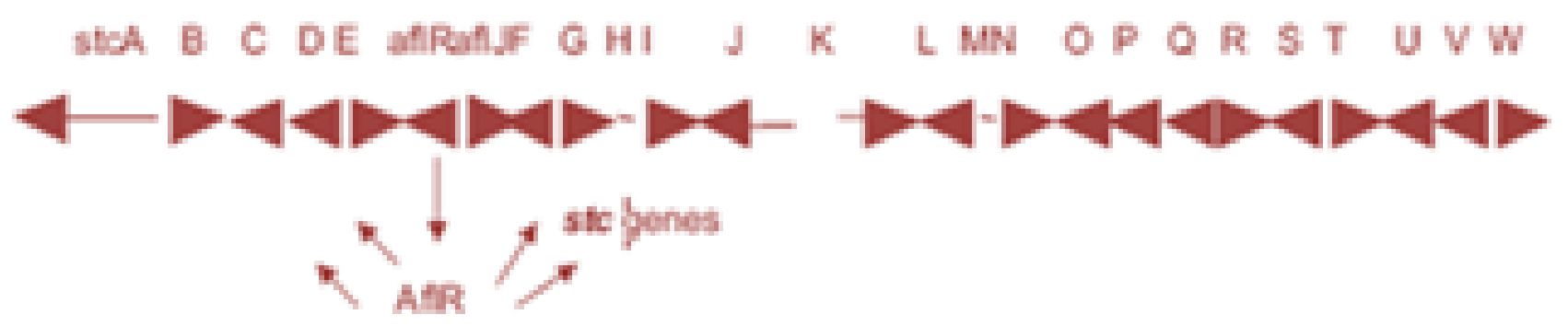

Figure I AfIR and afl are regulatory genes. The positively acting transcriptional factor is afIR requisite for the expression of the stc genes. ${ }^{\text {IS }}$

\section{Temperature and nitrogen effect on aflatoxin biosynthesis}

There are several biotic and abiotic environmental factors influence aflatoxin biosynthesis. ${ }^{22}$ According to the temperature and nitrogen effect on aflatoxin biosynthesis afR play key role. Nitrate play a suppressive effect on aflatoxin production, also over expression of the aflR gene by extra copies of afR overcomes and negative regulatory outcome on gene transcription of aflatoxin pathway. Aflatoxin formation is exactly affected by temperature. ${ }^{23}$ RT-PCR analyzed enough quantity of transcripts of commonly regulatory genes aflR and aflS. $^{23}$

\section{Conclusion}

The aflR sequence identified in these studies aflR gene cloning, expression and its pathogenicity of aflatoxin in human. We learn focus on the mechanisms of aflatoxin biosynthesis, we need to examine its regulatory mechanisms. The regulation of aflatoxin gene expression occurs at numerous levels and by several regulatory components. The nutritional and environmental factors effect on aflatoxin formation through the presence of $A f R$. The regulated expression of aflR also occurs in A. oryzae, which is generally used in the food industry. We expect that examination will lead to a finding of genes and molecules involved in pathogenicity and fungal continued existence that may give efforts to control diseases.

\section{Acknowledgements}

None.

\section{Conflict of interest}

The author declares no conflict of interest.

\section{References}

1. Lugauskas A, Krikštaponis A. Filamentous fungi isolated in hospitals and some medical institutions in Lithuania. Indoor and built environment. 2004;13(2):101-108.

2. Yu J, Thomas EC, William CN, et al. Aspergillus flavus genomics: gateway to human and animal health, food safety, and crop resistance to diseases. Revista iberoamericana de micología. 2005;22(4):194-202.

3. Burger G, Strauss J, Scazzocchio C, et al. nirA, the pathway-specific regulatory gene of nitrate assimilation in Aspergillus nidulans, encodes a putative GAL4-type zinc finger protein and contains four introns in highly conserved regions. Molecular and cellular biology. 1991;11(11):5746-5755.

4. Bennett JW, Rubin PL, Lee LS, et al. Influence of trace elements and nitrogen sources on versicolorin production by a mutant strain of Aspergillus parasiticus. Mycopathologia. 1979;69(3):161-166.

5. Chang PK, Ehrlich KC, Yu J, et al. Increased expression of Aspergillus parasiticus aflR, encoding a sequence-specific DNA-binding protein, relieves nitrate inhibition of aflatoxin biosynthesis. Appl Environ Microbiol. 1995;61(6):2372-2377.

6. Wen Y, Hidemi H, Hatsue A, et al. Function of the cypX and moxY genes in aflatoxin biosynthesis in Aspergillus parasiticus. Applied and environmental microbiology. 2005;71(6):3192-3198.

7. Woloshuk CP, Foutz KR, Brewer JF, et al. Molecular characterization of aflR, a regulatory locus for aflatoxin biosynthesis. Applied and Environmental Microbiology. 1994;60(7):2408-2414. 
8. Ehrlich KC, Cary JW, Ehrlich M. A broad bean cDNA clone encoding a DNA-binding protein resembling mammalian CREB in its sequence specificity and DNA methylation sensitivity. Gene. 1992;117(2):169178 .

9. Bhatnagar D, Ehrlich KC, Cleveland TE. Molecular genetic analysis and regulation of aflatoxin biosynthesis. Appl Microbiol Biotechnol. 2003;61(2):83-93.

10. Dhawale SS, Lane AC. Compilation of sequence-specific DNA-binding proteins implicated in transcriptional control in fungi. Nucleic acids research. 1993;21(24):5537-5546.

11. Payne GA, Nystrom GJ, Bhatnagar D, et al. Cloning of the afl-2 gene involved in aflatoxin biosynthesis from Aspergillus flavus. Appl Environ Microbio. 1993;59(1):156-162.

12. Laughon A, Gesteland RF. Primary structure of the Saccharomyces cerevisiae GAL4 gene. Mol Cell Biol. 1984;4(2):260-267.

13. Jiujiang Yu, Perng-Kuang Chang, Kenneth C, et al. Clustered pathway genes in aflatoxin biosynthesis. Applied and environmental microbiology. 2004;70(3):1253-1262.

14. Yuan GF, Fu YH, Marzluf GA. nit-4, a pathway-specific regulatory gene of Neurospora crassa, encodes a protein with a putative binuclear zinc DNA-binding domain. Mol Cell Biol. 1991;11(11):5735-5745.

15. Chang PK, Cary JW, Bhatnagar D, et al. Cloning of the Aspergillus parasiticus apa-2 gene associated with the regulation of aflatoxin biosynthesis. Appl Environ Microbiol. 1993;59(10):3273-3279.
16. Karakousis A, Tan L, Ellis D, et al. An assessment of the efficiency of fungal DNA extraction methods for maximizing the detection of medically important fungi using PCR. J Microbiol Methods. 2006;65(1):38-48.

17. Golemboski DB, Lomonossoff GP, Zaitlin M. Plants transformed with a tobacco mosaic virus nonstructural gene sequence are resistant to the virus. Proc Natl Acad Sci USA. 1990;87(16):6311-6315.

18. Engler-Blum G, Meier M, Frank J, et al. Reduction of background problems in nonradioactive Northern and Southern blot analyses enables higher sensitivity than 32P-based hybridizations. Analytical biochemistry. 1993;210(2):235-244.

19. Funk CD, Funk LB, Kennedy ME, et al. Human platelet/erythroleukemia cell prostaglandin $\mathrm{G} / \mathrm{H}$ synthase: cDNA cloning, expression, and gene chromosomal assignment. The FASEB Journal. 1991;5(9):2304-2312.

20. Yu J, Cary JW, Bhatnagar D, et al. Cloning and characterization of a cDNA from Aspergillus parasiticus encoding an $\mathrm{O}-$-methyltransferase involved in aflatoxin biosynthesis. Applied and environmental microbiology. 1993;59(11):3564-3571.

21. Hershey HP. External regulation of gene expression. Google Patents. 1997.

22. Demain AL. Induction of microbial secondary metabolism. Int Microbiol. 1998;1(4):259-264.

23. O'Brian GR, Georgianna DR, Wilkinson JR, et al. The effect of elevated temperature on gene transcription and aflatoxin biosynthesis. Mycologia. 2007;99(2):232-239. 